\title{
Clinical Study \\ Results in Assisted Peritoneal Dialysis: A Ten-Year Experience
}

\author{
Sara Querido, ${ }^{1}$ Patrícia Quadros Branco, ${ }^{2}$ Elisabete Costa, ${ }^{2}$ Sara Pereira, ${ }^{2}$ \\ Maria Augusta Gaspar, ${ }^{2}$ and José Diogo Barata ${ }^{2}$ \\ ${ }^{1}$ Department of Nephrology Centro Hospitalar do Médio Tejo, Avenida Xanana Gusmão, Apartado 45, \\ 2350-754 Torres Novas, Portugal \\ ${ }^{2}$ Department of Nephrology, Centro Hospitalar de Lisboa Ocidental, Carnaxide, Portugal
}

Correspondence should be addressed to Sara Querido; saraqueridoconde@gmail.com

Received 25 May 2015; Accepted 10 September 2015

Academic Editor: Hermann G. Haller

Copyright (C) 2015 Sara Querido et al. This is an open access article distributed under the Creative Commons Attribution License, which permits unrestricted use, distribution, and reproduction in any medium, provided the original work is properly cited.

\begin{abstract}
Background/Aims. Peritoneal dialysis is a successful renal replacement therapy (RRT) for old and dependent patients. We evaluated the clinical outcomes of an assisted peritoneal dialysis (aPD) program developed in a Portuguese center. Methods. Retrospective study based on 200 adult incident patients admitted during ten years to a PD program. We included all 17 patients who were under aPD and analysed various parameters, including complications with the technique, hospitalizations, and patient and technique survival. Results. The global peritonitis rate was lower in helped than in nonhelped patients: 0.4 versus 0.59 episodes/patient/year. The global hospitalization rate was higher in helped than in nonhelped patients: 0.67 versus 0.45 episodes/patient/year $(p=\mathrm{NS})$. Technique survival in helped patients versus nonhelped patients was $92.3 \%, 92.3 \%, 83.1 \%$, and $72.7 \%$ versus $91.9 \%$, $81.7 \%$, and $72.1 \%$, and $68.3 \%$, at $1,2,3$, and 4 years, respectively ( $p=$ NS), and patient survival in helped patients versus nonhelped patients was $93.3 \%$, $93.3 \%, 93.3 \%$, and $74.7 \%$ versus $95.9 \% 93.7 \%, 89 \%$, and $82 \%$ at $1,2,3$, and 4 years, respectively $(p=$ NS). Conclusions. aPD offers an opportune, reliable, and effective home care alternative for patients with no other RRT options.
\end{abstract}

\section{Introduction}

In the last two decades, most developed countries have seen a continuous growth in the number of patients with endstage renal disease (ESRD) commencing renal replacement therapy (RRT). It is possible to identify the main factors which influence this growth: aging of the population due to a greater life expectancy; increase in the incidence of chronic kidney disease related to age; better care of patients with chronic diseases; patients with physical limitations who survive now for longer periods of time; and developments in industry and biotechnology [1, 2]. In Portugal in 2014, almost $60 \%$ of patients starting dialysis were over the age of 65 years and only $8,73 \%$ of all incident patients started PD. Despite this increase in the number of elderly and dependent patients who need RRT [3], a decline in the utilization of peritoneal dialysis (PD) has occurred in a number of countries since the mid-1990s [4]. This decline is particularly acute for the elderly population [5]. Nevertheless, elderly and dependent patients benefit, specially, from PD as it would avoid travelling to dialysis centers, reduce hemodynamic instability
[6], diminish the risk of central venous catheter-associated bacteremia [5], improve blood pressure control [5, 7], and diminish the bacterial translocation and myocardial stunning [8]. Such a population cohort is susceptible to several physical barriers (decreased strength to lift PD bags, decreased manual dexterity, and decreased vision, mobility, and hearing) and cognitive barriers (language, noncompliance, dementia, and psychiatric conditions). Thus, providing home care assistance to support those patients on PD may help increasing the number of individuals that can be safely treated at home [9] as well as reducing hazards related to personal limitations. Assistance to PD patients involves the identification and training of an individual (other than the patient) to perform dialysis-related tasks, such as connecting the patient to the cycler, setting up the cycler, disconnecting the patient from the cycler, or performing continuous ambulatory peritoneal dialysis (CAPD) exchanges. Since 1997 [10], following the publication of the first successful French experience (when home care nurses treated elderly patients with assisted CAPD) assisted PD (aPD) became a valuable alternative to 
provide successful RRT for old and dependent patients. Over the past decade, 11,557 patients started PD in France, out of which $44.6 \%$ have been on aPD [11]. The nurses who assist at home in France are paid directly by patients, who are partially reimbursed by the French healthcare insurance. Elderly patients are also successfully treated with PD in Hong Kong, where PD is the first treatment option. In March 2007, $80 \%$ of patients (median age: 62.3 years) were on PD [12]. Presently aPD is a dialysis modality in evolution all over Western Europe, Canada, South America, and Asia [11-16].

Although aPD is a valuable and successful renal replacement therapy for old and dependent patients we have to consider and face the lack of social support for patients in our country. The aim of this study was to evaluate the results of the aPD program, offered as first option or last resort to elderly or physically incapable end-stage renal disease patients, considering the clinical outcomes of this technique in a single Portuguese center.

\section{Material and Methods}

This is a retrospective study performed at a single PD Unity in Portugal (PD Unity of Hospital de Santa Cruz, Carnaxide, Portugal), based on the study of 200 adult incident patients admitted during 10 years (2004-2014) to the PD program. We included and studied a total of 17 patients with physical or cognitive debilities who were under aPD. Assisted-care patients were defined as patients who are unable to perform peritoneal fluid exchange at the beginning of PD or who lack the ability to perform their own treatment and have, therefore, to rely on nonprofessional care, including family members or domestic workers. We analyzed demographic, clinical, and laboratory parameters, complications with the technique, hospitalizations, and patient and technique survival through research in clinical processes. The degree of dependence was analyzed through the application of the Davies Score [17] and Karnofsky Index. Normally distributed variables were expressed as mean \pm standard deviation and nonnormally distributed variables were expressed as frequency and percentage. Unadjusted analysis was performed by the KaplanMeier method to analyse technique survival between self-care and aPD patients. Technique failure classified the dropout from aPD to Hemodialysis due to peritoneal membrane failure or peritonitis. The technique survival was defined in patients who remained on aPD during the observation period and kidney receptors allograft and patients who died during the $\mathrm{aPD}$ program due to any reason other than peritonitis or peritoneal membrane failure.

All statistical tests were performed using the Statistical Package for the Social Sciences (SPSS) 14.0 software (SPSS, Inc., Chicago, IL, USA). Categorical variables were described as numbers or percentage of relative frequencies and quantitative variables as mean \pm standard deviation (SD) for continuous normally distributed variables. Cox regression was used to compare survival rates.

Differences between clinical data were assessed by Student's $t$-test for paired samples for normal variables and paired Wilcoxon test for continuous data with nonnormal distribution. A $p$ value of $<0.05$ was considered to be statistically significant.

\section{Results}

We followed a cohort of 17 consecutive incident patients who were engaged in aPD from January 2004 to October 2014. Median age was $58 \pm 20$ years; 9 patients were men, 12 patients had hypertension, and 6 had diabetes. Fourteen patients had only one helper, like a close relative; 2 patients were treated by multiple family members; and one patient received treatment from 2 home assistance employees. One patient was on $\mathrm{PD}$ due to vascular access failure; 5 patients chose PD; and for 11 patients PD was a family's choice. Five patients had physical and cognitive limitations ab initium. The mean age of the ones who had physical limitations was 35.2 years; patients with cognitive limitations had a mean age of 65 years; 7 patients were treated with automated assisted peritoneal dialysis (APD) and 10 patients with CAPD. The Davies Score was greater than 2 in $52.9 \%$; Karnofsky Index was less than 70 in $64.7 \%$. The patients were under PD for $36.98 \pm 31.43$ months; 4 of them had an acute onset of the technique; kt/V weekly was $2.22 \pm 0.60$ and nPCR was $0.88 \pm 0.30 \mathrm{~g} / \mathrm{Kg} /$ day. Peritoneal equilibration test (PET) was performed in 14 patients: 8 were low-average and 6 were high-average transporters. The residual renal function was $3.02 \pm 3.85 \mathrm{~mL} / \mathrm{min} / 1.73 \mathrm{~m}^{2}$ and 3 patients were anuric. Demographic and PD related parameters in patients under autonomous PD and aPD are compared in Table 1.

Half of the patients have never had a peritonitis episode; 2 patients had a tunnel infection; and 9 patients had one or more episodes of exit-site infection. Six patients needed more than 1 Tenckhoff catheter; 4 patients died during PD technique; 3 patients started haemodialysis (1 due to PD membrane failure and 2 due to peritonitis); and 1 patient received a kidney allograft. The global peritonitis rate was lower in helped than in nonhelped patients: 0.4 versus 0.59 episodes/patient/year. The global hospitalization rate was higher in helped than in nonhelped patients: 0.67 versus 0.45 episodes/patient/year ( $p=$ NS). Technique survival in helped patients versus nonhelped patients was $92,3 \%, 92.3 \%, 83,1 \%$, and $72.7 \%$ versus $91,9 \%, 81,7 \%, 72,1 \%$, and $68,3 \%$, at $1,2,3$, and 4 years, respectively ( $p=$ NS) (Figure 1) and patient survival in helped patients versus nonhelped patients was $93,3 \%, 93,3 \%, 93,3 \%$, and $74,7 \%$ versus $95,9 \% 93,7 \%, 89 \%$, and $82 \%$ at $1,2,3$, and 4 years, respectively ( $p=N S$ ) (Figure 2 ). Two patients remained on aPD for more than 7 years.

\section{Discussion}

The outcome of PD is usually assessed by patient survival, technique survival, and peritonitis incidence [18]. PD shows no difference in patient survival, technique survival, and peritonitis rate [19], between elderly and younger patients. Nonetheless, the outcome of assisted-care and self-care in elderly PD patients is not consistent. The RDPLF report showed that patients under assisted-care, either by family 
TABLE 1: Demographic and PD related parameters in patients under autonomous PD and aPD.

\begin{tabular}{|c|c|c|c|c|}
\hline & \multicolumn{2}{|c|}{ Autonomous PD $(n=183)$} & \multicolumn{2}{|c|}{ Assisted PD $(n=17)$} \\
\hline Age (years) & \multicolumn{2}{|c|}{$55,7 \pm 15,2$} & \multicolumn{2}{|c|}{$58 \pm 20$} \\
\hline Male $(n / \%)$ & \multicolumn{2}{|c|}{$122(66,67)$} & \multicolumn{2}{|c|}{$9(52,94)$} \\
\hline Hypertension $(n / \%)$ & \multicolumn{2}{|c|}{$172(93,99)$} & \multicolumn{2}{|c|}{$12(70,59)$} \\
\hline Diabetes $(n / \%)$ & \multicolumn{2}{|c|}{$58(31,69)$} & \multicolumn{2}{|c|}{$6(35,29)$} \\
\hline $\operatorname{APD}(n / \%)$ & \multicolumn{2}{|c|}{$64(34,97)$} & \multicolumn{2}{|c|}{$7(41,18)$} \\
\hline CAPD $(n / \%)$ & \multicolumn{2}{|c|}{$119(65,03)$} & \multicolumn{2}{|c|}{$10(58,82)$} \\
\hline Time under PD (months) & \multicolumn{2}{|c|}{$29,7 \pm 22,7$} & \multicolumn{2}{|c|}{$36,98 \pm 31,43$} \\
\hline \multirow{4}{*}{ Technique survival (months/\%) } & $12 \mathrm{~m}$ & 91,9 & $12 \mathrm{~m}$ & 92,3 \\
\hline & $24 \mathrm{~m}$ & 81,7 & $24 \mathrm{~m}$ & 92,3 \\
\hline & $36 \mathrm{~m}$ & 72,1 & $36 \mathrm{~m}$ & 83,1 \\
\hline & $48 \mathrm{~m}$ & 68,3 & $48 \mathrm{~m}$ & 72,7 \\
\hline \multirow{4}{*}{ Patient survival (months/\%) } & $12 \mathrm{~m}$ & 95,9 & $12 \mathrm{~m}$ & 93,3 \\
\hline & $24 \mathrm{~m}$ & 93,7 & $24 \mathrm{~m}$ & 93,3 \\
\hline & $36 \mathrm{~m}$ & 89 & $36 \mathrm{~m}$ & 93,3 \\
\hline & $48 \mathrm{~m}$ & 82 & $48 \mathrm{~m}$ & 74,7 \\
\hline Weekly kt/V & \multicolumn{2}{|c|}{$2,4 \pm 0,70$} & \multicolumn{2}{|c|}{$2,22 \pm 0,60$} \\
\hline nPCR (g/Kg/day) & \multicolumn{2}{|c|}{0,93} & \multicolumn{2}{|c|}{$0,88 \pm 0,30$} \\
\hline \multirow{4}{*}{ PET } & \multirow{4}{*}{$N=175$} & $\mathrm{D} / \mathrm{P}<0,5=2 \%$ & \multirow{2}{*}{\multicolumn{2}{|c|}{$\mathrm{D} / \mathrm{P} 0,5-0,64=8$}} \\
\hline & & $\mathrm{D} / \mathrm{P} 0,5-0,64=35 \%$ & & \\
\hline & & $\mathrm{D} / \mathrm{P} 0,65-0,81=62 \%$ & & $\mathrm{D} / \mathrm{P} 0,65-0,81=6$ \\
\hline & & $\mathrm{D} / \mathrm{P}>0,81=1 \%$ & & \\
\hline Residual renal function $\left(\mathrm{mL} / \mathrm{min} / 1,73 \mathrm{~m}^{2}\right)$ & $N=155$ & $7,14 \pm 11$ & $N=14$ & $3,02 \pm 3,85$ \\
\hline
\end{tabular}

APD: automated peritoneal dialysis; CAPD: continuous ambulatory peritoneal dialysis; PET: peritoneal equilibration test.

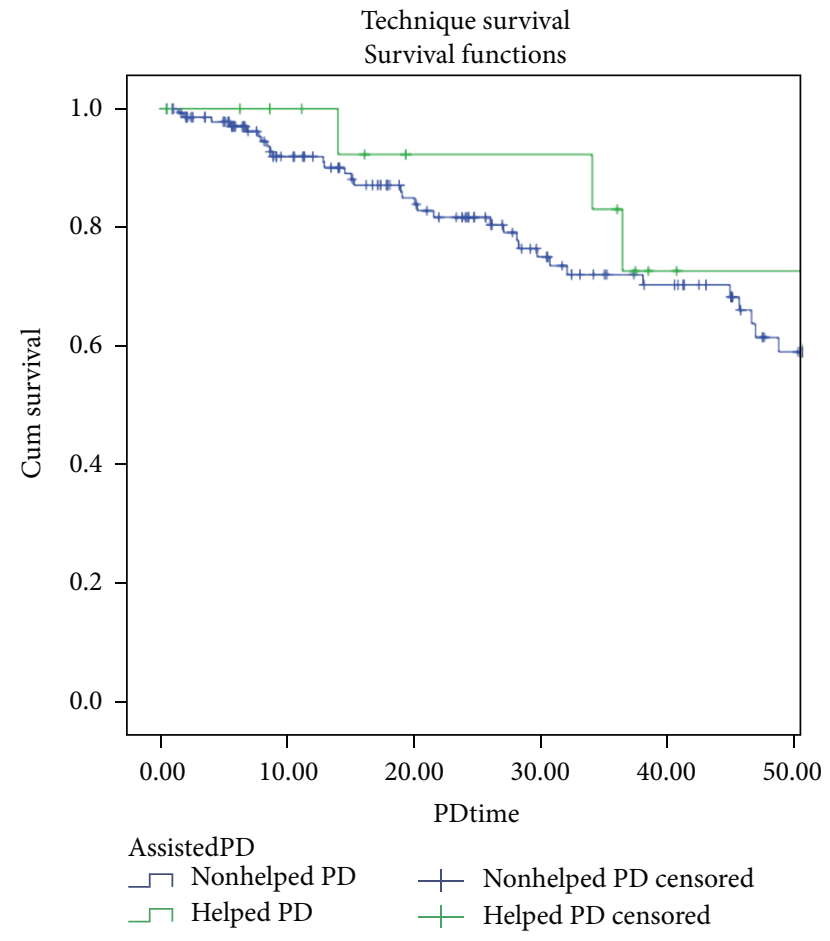

FIGURE 1: Technique survival in helped and nonhelped patients. members or by nurses, had a poorer survival rate than selfcare patients [9]. On the other hand, in a Hong Kong research, no significant differences were found in self-care elderly and nonelderly patients in terms of survival and technique survival. Our study showed that assisted-care PD patients had a poorer outcome in terms of patient survival (12th, 24 th, and 48th months) and hospitalization rate but a better performance in terms of peritonitis incidence and technique survival. Lobbedez et al. [11] studied $36 \mathrm{aPD}$ patients and observed a relatively high peritonitis rate, with $50 \%$ presenting at least 1 episode per year. Issad et al. showed that peritonitis and exit-site infection rates were not significantly different between aPD and self-care PD patients [10]. Verger et al. revealed that the probability of being peritonitis-free at 2 years was higher for patients assisted by a family member than for those assisted by a private nurse [14]. In our study, the global peritonitis rate was lower in helped than in nonhelped patients (0,4 versus 0,59 episodes/patient/year). As in Verger et al. study, this fact could be explained by the high dedication level of family members.

According to Lobbedez et al. [11] a higher percentage of aPD patients (79\%) were hospitalized during the first followup year, with peritonitis being the most frequent cause of hospitalization. Our results are similar to the literature with a global hospitalization rate higher in helped than in nonhelped 


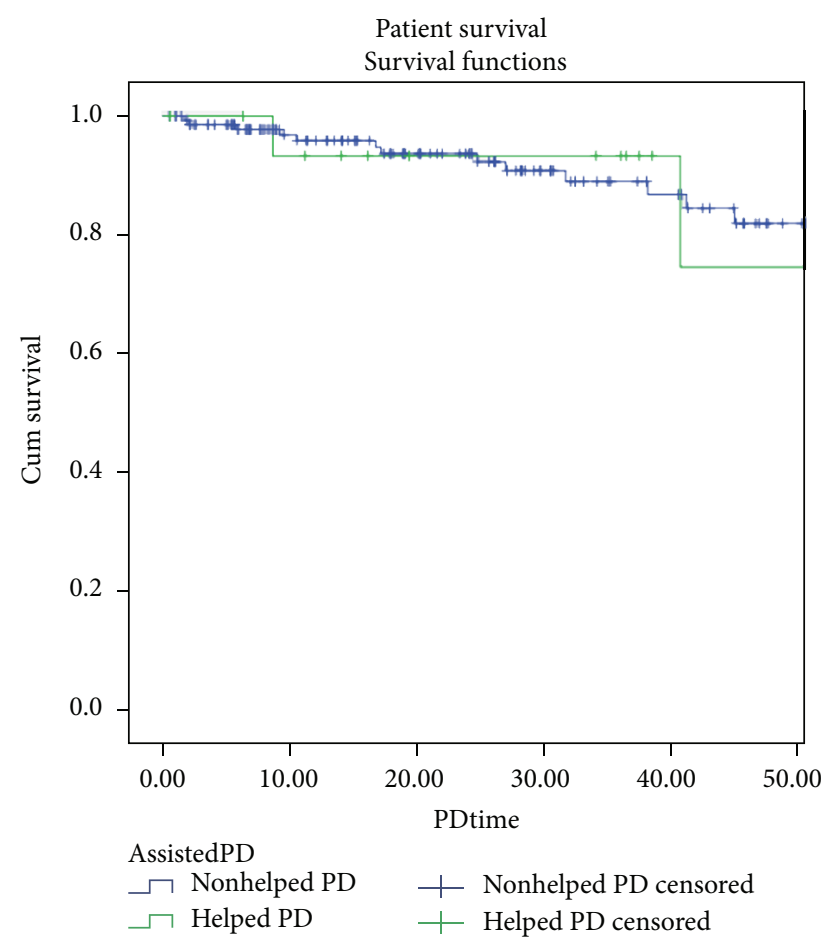

FIGURE 2: Patient survival in helped and nonhelped patients.

patients [0.67 versus 0.45 episodes/patient/year $(p=N S)$ ]. This fact could be explained by the high comorbidity index in the aPD patients.

There are not many studies concerning technique survival on assistance method $[9,18]$. Those studies showed no association between technique survival and assistance method. We found that technique survival was better in aPD patients. It is difficult to interpret the facts due to the small number of patients in aPD program, but, globally, aPD did not show any disadvantage in terms of technique survival. Lobbedez et al. [11] reported $83 \%$ 1-year survival of aPD patients at their center. Povlsen et al. [16] showed that the 1-year and 2year survival rates of functionally dependent elderly patients on aPD were $58 \%$ and $48 \%$, respectively. In our study, the survival rate was $93,3 \%$ in the first 3 years under technique, with 2 patients remained on aPD for more than 7 years. The patient who died earlier was a dependent patient with a severe heart disease. Nevertheless, in our series, the survival rate in aPD patients is probably overestimated, considering that these patients had a median age of 58 years, much younger when compared with aPD patients from other series [10, 15].

In UK, aPD is in its infancy. There is no extra funding for providing assistance, so developing a service depends on local enthusiasm. The model of care being developed is based on aPD with one visit per day from a paid carer and the patient or family carrying out the connection and disconnection to/from machine. The community nursing service is not adequately staffed or funded to take on this extra role [20].

\section{Conclusions}

Our results compare favourably with international reports. In this clinical observation study, aPD offered an opportune, reliable, and effective home care alternative for patients with no other renal replacement therapy options. Due do the lack of support from social institutions the helpers were close relatives in almost every case. It is necessary to adopt measures and institutional support to care for these patients, not forgetting that some of them are young people with a considerable life expectancy, despite cognitive or motor deficits. Nevertheless, larger, longer, and better studies on aPD are warranted. Till now, studies have showed that assistance gives dependent patients an opportunity to have a home-based dialysis modality, increasing the number of patients who can choose an appropriate treatment despite their physical, cognitive, and social conditions.

\section{Consent}

Informed consent was obtained from all individual participants included in the study.

\section{Conflict of Interests}

The authors declare that there is no conflict of interests regarding the publication of this paper.

\section{References}

[1] National Institute of Diabetes and Digestive and Kidney Diseases, "United States renal data system 2007 annual data report: atlas of chronic kidney disease \& end-stage renal disease in the United States," American Journal of Kidney Diseases, vol. 51, supplement 1, pp. S82-S98, 2008.

[2] K. J. Jagger, P. C. W. van Dijk, F. W. Dekker, B. Stengel, K. Simpson, and J. D. Briggs, "The epidemic of aging in renal replacement therapy: an update on elderly patients and their outcomes," Clinical Nephrology, vol. 60, no. 5, pp. 352-360, 2003.

[3] E. A. Brown and L. Johansson, "Dialysis options for end-stage renal disease in older people," Nephron: Clinical Practice, vol. 119, supplement 1, pp. c10-c13, 2011.

[4] P. Dalal, H. Sangha, and K. Chaudhary, "In peritoneal dialysis, is there sufficient evidence to make 'PD first' therapy?" International Journal of Nephrology, vol. 2011, Article ID 239515, 5 pages, 2011.

[5] P. G. Blake, "Peritoneal dialysis-a 'kinder, gentler' treatment for the elderly?" Peritoneal Dialysis International, vol. 28, no. 5, pp. 435-436, 2008.

[6] E. A. Brown, L. Johansson, K. Farrington et al., "Broadening Options for Long-term Dialysis in the Elderly (BOLDE): differences in quality of life on peritoneal dialysis compared to haemodialysis for older patients," Nephrology Dialysis Transplantation, vol. 25, no. 11, pp. 3755-3763, 2010.

[7] N. Dimkovic, V. Aggarwal, S. Khan, M. Chu, J. Bargman, and D. G. Oreopoulos, "Assisted peritoneal dialysis: what is it and who does it involve?" Advances in Peritoneal Dialysis, vol. 25, pp. 165-170, 2009. 
[8] N. M. Selby and C. W. McIntyre, "Peritoneal dialysis is not associated with myocardial stunning," Peritoneal Dialysis International, vol. 31, no. 1, pp. 27-33, 2011.

[9] C. Castrale, D. Evans, C. Verger et al., "Peritoneal dialysis in elderly patients: report from the French peritoneal dialysis registry (RDPLF)," Nephrology Dialysis Transplantation, vol. 25, no. 1, pp. 255-262, 2010.

[10] B. Issad, D. Benevent, M. Allouache et al., "213 Elderly uremic patients over 75 years of age treated with long-term peritoneal dialysis: a French multicenter study," Peritoneal Dialysis International, vol. 16, supplement 1, pp. S414-S418, 1996.

[11] T. Lobbedez, R. Moldovan, M. Lecame, B. H. de Ligny, W. El Haggan, and J.-P. Ryckelynck, "Assisted peritoneal dialysis. Experience in a French renal department," Peritoneal Dialysis International, vol. 26, no. 6, pp. 671-676, 2006.

[12] P. K.-T. Li and C.-C. Szeto, "Success of the peritoneal dialysis programme in Hong Kong," Nephrology Dialysis Transplantation, vol. 23, no. 5, pp. 1475-1478, 2008.

[13] P.-Y. Durand and C. Verger, "The state of peritoneal dialysis in France," Peritoneal Dialysis International, vol. 26, no. 6, pp. 654657, 2006.

[14] C. Verger, M. Duman, P.-Y. Durand, G. Veniez, E. Fabre, and J.-P. Ryckelynck, "Influence of autonomy and type of home assistance on the prevention of peritonitis in assisted automated peritoneal dialysis patients. An analysis of data from the French Language Peritoneal Dialysis Registry," Nephrology Dialysis Transplantation, vol. 22, no. 4, pp. 1218-1223, 2007.

[15] D. Paulsen, J. Kronborg, and K. Solbakken, "Organization of peritoneal dialysis in Oppland County, Norway," Peritoneal Dialysis International, vol. 26, supplement 2, p. S124, 2006.

[16] J. V. Povlsen, K. Lomholt, and P. Ivarsen, "Assisted APD (aAPD) for the elderly patient dependent on a carer," Peritoneal Dialysis International, vol. 26, supplement 2, p. S110, 2006.

[17] S. J. Davies, L. Russell, J. Bryan, L. Phillips, and G. I. Russell, "Comorbidity, urea kinetics, and appetite in continuous ambulatory peritoneal dialysis patients: their interrelationship and prediction of survival," American Journal of Kidney Diseases, vol. 26, no. 2, pp. 353-361, 1995.

[18] X. Yang, W. Fang, J. Kothari et al., "Clinical outcomes of elderly patients undergoing chronic peritoneal dialysis: experiences from one center and a review of the literature," International Urology and Nephrology, vol. 39, no. 4, pp. 1295-1302, 2007.

[19] P. K.-T. Li, M. C. Law, K. M. Chow et al., "Good patient and technique survival in elderly patients on continuous ambulatory peritoneal dialysis," Peritoneal Dialysis International, vol. 27, supplement 2, pp. S196-S201, 2007.

[20] E. A. Brown, "How to address barriers to peritoneal dialysis in the elderly," Peritoneal Dialysis International, vol. 31, supplement 2, pp. S83-S85, 2011. 


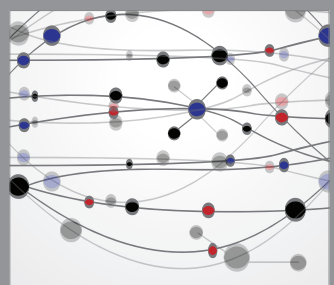

The Scientific World Journal
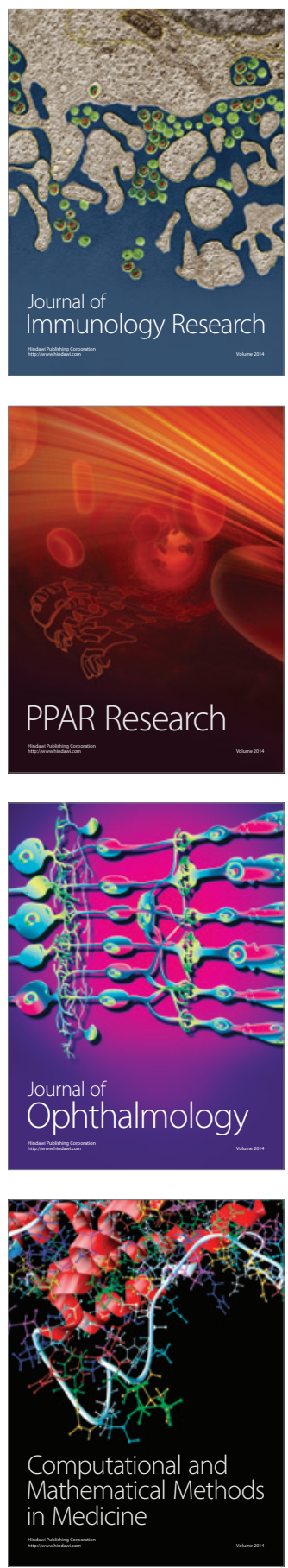

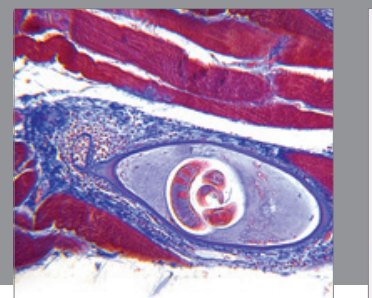

Gastroenterology

Research and Practice
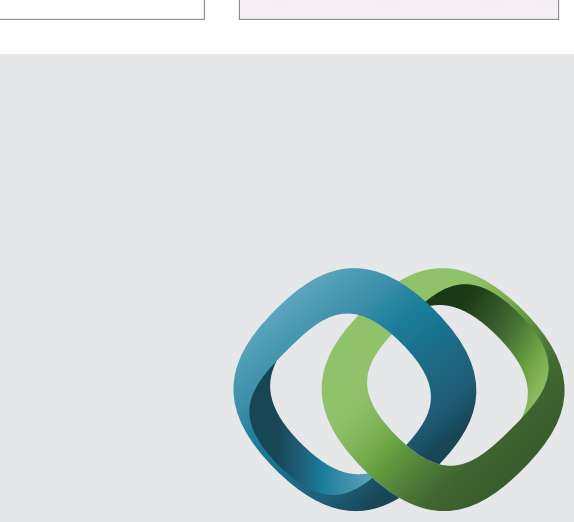

\section{Hindawi}

Submit your manuscripts at

http://www.hindawi.com
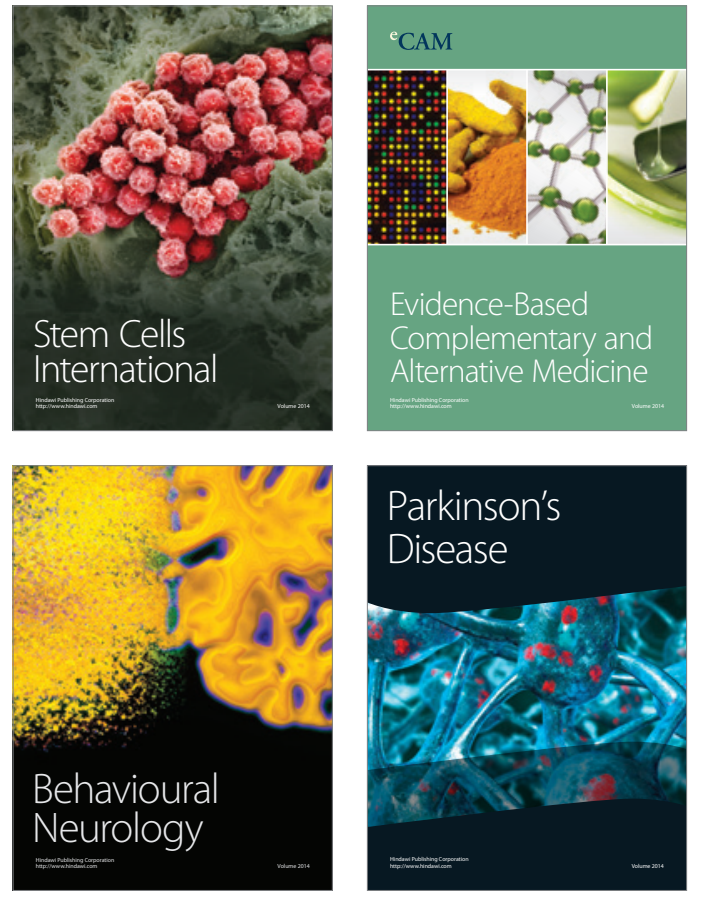
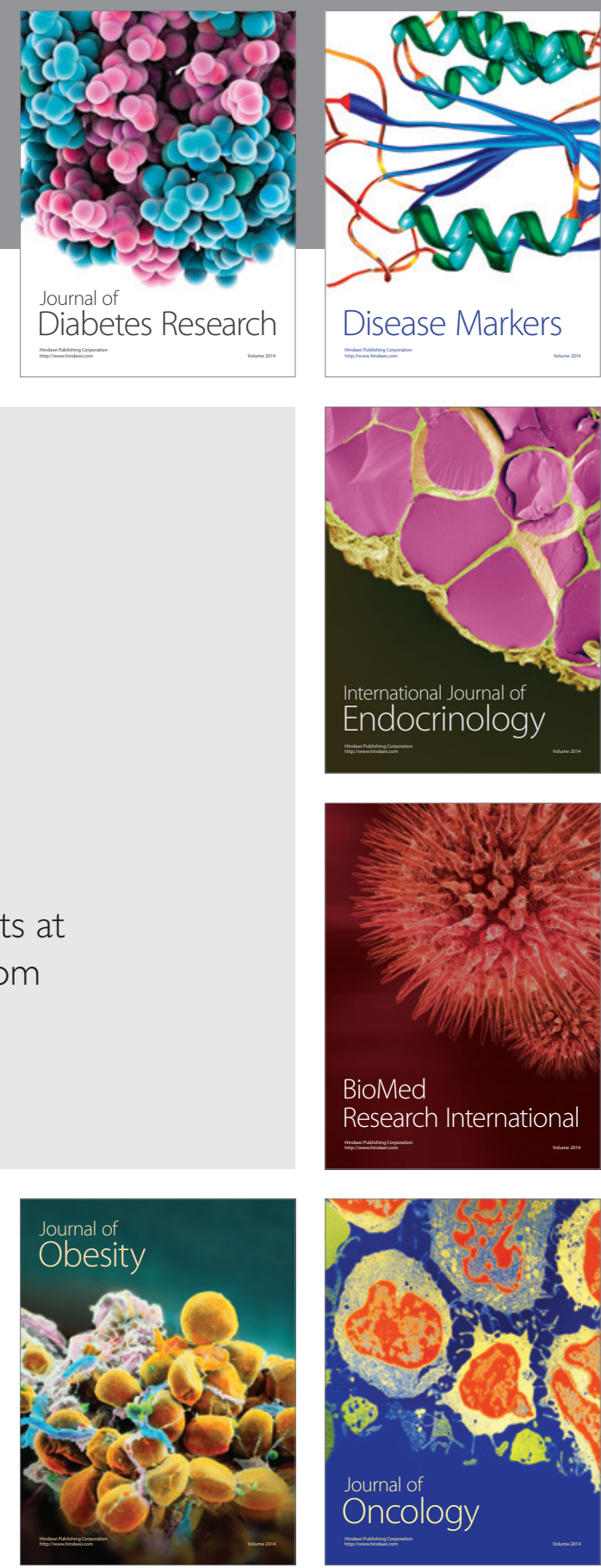

Disease Markers
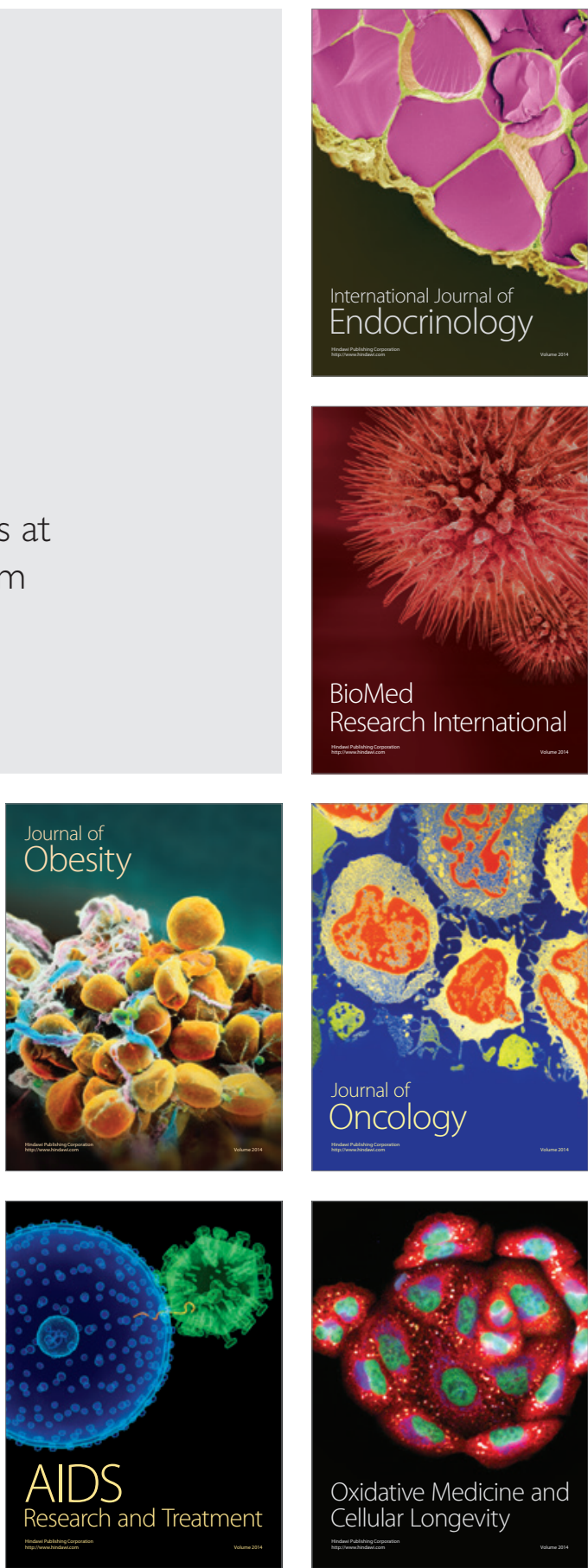